\title{
Seletividade dos herbicidas nicosulfuron e foramsulfuron + iodosulfuron methyl sodium a diferentes cultivares de milho
}

\section{Selectivity of nicosulfuron and foramsulfuron + iodosulfuron methyl sodium to different corn cultivars}

\author{
Robinson Luiz Contiero ${ }^{1}$
}

\section{Resumo}

O objetivo do presente trabalho foi avaliar a seletividade dos herbicidas nicosulfuron e a mistura de foramsulfuron + iodosulfuron methyl sodium a diferentes híbridos de milho Agroeste (AS 1545, AS 1548, S2 709, AS 32, AS 3466 e AS 1533). O delineamento experimental utilizado foi o de blocos ao acaso, com 7 tratamentos e 5 repetições para cada híbrido. As doses dos herbicidas (em g i.a. ha ${ }^{-1}$ ) foram: foramsulfuron + iodosulfuron methyl sodium: $22,5+1,5 ; 45,0+3,0$ e 90,0 + 6,0 e nicosulfuron: 30,60 e 90. Avaliou-se a fitotoxicidade, além de parâmetros relativos aos componentes de produção da cultura e produtividade. Os cultivares de milho testados possuem tolerância diferencial aos herbicidas utilizados; o híbrido S2 709 teve sua produtividade diminuída quando o herbicida foramsulfuron + iodosulfuron methyl sodium foi aplicado nas doses de $45,0+3,0$ e $90,0+6,0$ g i.a. ha ${ }^{-1}$; o híbrido AS 1533 teve sua produtividade reduzida quando os herbicidas foramsulfuron + iodosulfuron methyl sodium e nicosulfuron foram aplicados nas doses de 90,0 +6,0 g i.a. ha ${ }^{-1}$ e $120 \mathrm{~g}$ i.a. ha ${ }^{-1}$, respectivamente.

Palavras-chave: Controle químico, fitotoxicidade, Sanson, Equip Plus, Zea mays

\begin{abstract}
This work aimed the evaluation of the selectivity of nicosulfuron and foramsulfuron + iodosulfuron methyl sodium herbicides to different Agroeste corn hybrids (AS 1545, AS 1548, S2 709, AS 32, AS 3466 and AS 1533). The trials were arranged in a randomized complete block design with 7 treatments and 5 replications for each hybrid. Herbicides rates (in g i.a. ha ${ }^{-1}$ ) were: foramsulfuron + iodosulfuron methyl sodium: $22.5+1.5 ; 45.0+3.0$ and $90.0+6.0$ and nicosulfuron: 30,60 and 90 . Phytotoxicity and the parameters relative to the components of crop production and yield were evaluated. The corn cultivars had differential tolerance to the herbicides; S2 709 hybrid had its yield decreased when foramsulfuron + iodosulfuron methyl sodium was applied at $45.0+3.0$ and $90.0+6.0$ g i.a. ha ${ }^{-1}$; the AS 1533 hybrid had its yield reduced when foramsulfuron + iodosulfuron methyl sodium and nicosulfuron were applied at $90.0+6.0$ g i.a. ha ${ }^{-1}$ and 120.0 g i.a. ha ${ }^{-1}$ respectively.
\end{abstract}

Key words: Chemical control, phytotoxic effect, Sanson. Equip Plus, Zea mays

\footnotetext{
1 Eng. Agr., Prof. Dr. do Departamento de Agronomia da Universidade Estadual de Maringá - UEM. Av.Colombo, 5790, 87.020900, Maringá/PR. Tel. (44) 3261-8917. E-mail: rcontiero@gmail.com.
} 


\section{Introdução}

O manejo de plantas daninhas é de grande importância para obtenção de uma boa produtividade, já que estas requerem para seu desenvolvimento, os mesmos fatores exigidos pela cultura do milho, ou seja, água, luz e nutriente, estabelecendo um processo competitivo quando cultura e plantas daninhas se desenvolvem conjuntamente.

Os efeitos negativos detectados no crescimento e produtividade da cultura do milho, devido à presença de plantas daninhas, já foram relatados por vários autores (CARVALHO; GALLI, 1993; VELINI et al., 1993; SILVA; ASMUS; CORREA, 1993; ROSSI et al., 1996, PINTO et al., 2000; CONSTANTIN et al., 2000). KARAM; MELHORANÇA (2000) relatam perdas da ordem de $85 \%$ em lavouras de milho onde não se utilizou nenhum método de controle das plantas daninhas. Relatam ainda, que as perdas médias na cultura do milho em função da presença de plantas daninhas são da ordem de 13,1\%.

Diversos são os herbicidas e/ou misturas recomendados para o controle de plantas daninhas em pré-emergência na cultura de milho (SILVA; MELHORANÇA, 1991). Com o aumento da área cultivada com milho no Brasil (CONAB, 2009), a adoção das tecnologias disponíveis e a utilização da semeadura direta, a utilização de herbicidas em pósemergência tem se tornado cada vez mais freqüente (SILVA; RODRIGUES; BEGLIOMINI, 1998).

Entre os herbicidas tradicionalmente utilizados para o controle de plantas daninhas em milho, encontram-se a atrazine e o nicosulfuron. A atrazine, do grupo das triazinas, controla plantas daninhas latifoliadas, algumas gramíneas anuais, sendo a cultura seletiva, podendo ser aplicada tanto em pré como em pós-emergência inicial (RODRIGUES; ALMEIDA, 1998; ANDREI, 2005; LORENZI, 2000).

A tolerância diferencial do milho aos herbicidas é importante fator que vem sendo estudado por diversos autores (DAMIÃO FILHO; MÔRO;
TAVEIRA， 1996; GREEN; ULRICH， 1994; GREEN, 1998). A inativação metabólica é a base da seletividade de muitos herbicidas comerciais, mas a modificação do sítio-alvo (local de atuação do herbicida na planta) em laboratório tem se tornado prática importante e crescente (NEWHOUSE; WANG; ANDERSON, 1991).

Há consideráveis evidências da tolerância diferencial de cultivares de milho aos herbicidas do grupo das sulfoniluréias, em especial ao nicosulfuron (DAMIÃO FILHO; MORO; TAVEIRA, 1996; GREEN; ULRICH, 1994). A EMBRAPA (2004) recomenda a realização de testes de seletividade antes da utilização de herbicidas no controle de plantas daninhas em lavouras de milho.

De acordo com (PEREIRA FILHO; OLIVEIRA; PIRES, 2000), que estudou a tolerância de híbridos de milho ao herbicida nicosulfuron, embora as avaliações de fitotoxicidade realizadas até 21 dias após semeadura não tenham restringido o uso do nicosulfuron nos híbridos estudados, o ensaio foi conduzido até a colheita, em que se avaliaram a altura da planta e espigas, o índice de espigas e a produção de grãos. A análise de variância não revelou efeito significativo para nenhum dos parâmetros estudados.

O trabalho realizado por (CARVALHO; PERUCHI; PALAZZO, 2001), com o híbrido de milho "Master" demonstrou que os herbicidas foramsulfuron + iodosulfuron methyl sodium $(36,0$ $+2,4$ e 45,0 + 3,0 g i.a. ha-1) e nicosulfuron (50 g i.a $\mathrm{ha}^{-1}$ ) foram eficientes no controle das plantas daninhas Bidens pilosa, Commelina benghalensis e Digitaria horizontalis e seletivos ao híbrido de milho utilizado.

Dessa forma, o objetivo do presente trabalho foi avaliar a seletividade dos herbicidas nicosulfuron e foramsulfuron + iodosulfuron methyl sodium a diferentes híbridos de milho. 


\section{Material e métodos}

Foram conduzidos seis experimentos na estação experimental da UNIOESTE, localizada no município de Marechal Cândido Rondon/PR, em solo classificado como "Latossolo Vermelho Eutroférrico" (EMBRAPA, 1999), com semeadura em 03 de dezembro de 2004 e colheita em abril de 2005, de acordo com o ciclo de cada híbrido.

O delineamento experimental utilizado foi o de blocos ao acaso, constituído de 7 tratamentos e 5 repetições para cada híbrido, em parcelas de $3,2 \mathrm{~m}$ de largura por 7,0 $\mathrm{m}$ de comprimento As parcelas foram compostas por quatro linhas, com espaçamento de 0,80 m entre linhas. Como área útil considerou-se as duas linhas centrais de cada parcela.

Os híbridos de milho utilizados nos experimentos foram: AS 1548 (híbrido simples super-precoce, finalidade grãos e silagem de grãos úmidos), AS 1545 (híbrido simples super-precoce, finalidade silagem de grãos), S2 709 (híbrido simples, precoce, finalidade grãos), AS 32 (híbrido duplo precoce, finalidade silagem de planta inteira), AS 3466 (híbrido triplo precoce, finalidade silagem de grãos úmidos) e AS 1533 (híbrido simples precoce, finalidade silagem de grão úmido), semeados em sistema de semeadura direta e espaçamento de 0,8 $\mathrm{m}$ entre linhas e população de 65.000 plantas ha $^{-1}$ para o híbrido AS 1548 e 60.000 plantas ha $^{-1}$ para os demais híbridos.

Os tratamentos utilizados no experimento estão descritos na Tabela 1 (RODRIGUES; ALMEIDA, 1998; ANDREI, 2005). Em todos os tratamentos realizou-se o controle manual das plantas daninhas com objetivo de evitar qualquer tipo de interferência destas plantas na cultura do milho.

Tabela 1. Tratamentos utilizados nos experimentos com a cultura do milho. Marechal Cândido Rondon/PR, $2004 / 2005$

\begin{tabular}{|c|c|c|c|c|c|c|c|c|}
\hline \multirow[t]{2}{*}{ Trat } & \multirow[t]{2}{*}{ Nome Comum } & \multirow{2}{*}{$\begin{array}{c}\text { Nome } \\
\text { Comercial }\end{array}$} & \multirow[t]{2}{*}{ Grupo químico } & \multicolumn{2}{|c|}{ Formulação } & \multicolumn{2}{|c|}{$\begin{array}{c}\text { Dose } \\
\left(\mathrm{L} / \mathrm{g} \mathrm{ha}^{-1}\right)\end{array}$} & \multirow[t]{2}{*}{ C.T } \\
\hline & & & & Tipo & Conc. & I.A. & P.C. & \\
\hline 1 & Testemunha & - & - & - & - & - & - & - \\
\hline 2 & $\begin{array}{l}\text { Foramsulfuron } \\
+ \text { Iodosulfuron- } \\
\text { Methyl-Sodium }\end{array}$ & Equip Plus & Sulfoniluréias & WG & $\begin{array}{c}300+20 \\
\mathrm{~g} \mathrm{~kg}^{-1}\end{array}$ & $\begin{array}{c}22,5+ \\
1,5\end{array}$ & 75 & III \\
\hline 3 & $\begin{array}{l}\text { Foramsulfuron } \\
+ \text { Iodosulfuron- } \\
\text { Methyl-Sodium }\end{array}$ & Equip Plus & Sulfoniluréias & WG & $\begin{array}{c}300+20 \\
\mathrm{~g} \mathrm{~kg}^{-1}\end{array}$ & $45+3,0$ & 150 & III \\
\hline 4 & $\begin{array}{l}\text { Foramsulfuron } \\
+ \text { Iodosulfuron- } \\
\text { Methyl-Sodium }\end{array}$ & Equip Plus & Sulfoniluréias & WG & $\begin{array}{c}300+20 \\
\mathrm{~g} \mathrm{~kg}^{-1}\end{array}$ & $90+6,0$ & 300 & III \\
\hline 5 & Nicosulfuron & $\begin{array}{c}\text { Sanson } 40 \\
\text { CS }\end{array}$ & Sulfoniluréias & $\mathrm{CS}$ & $40 \mathrm{~g} \mathrm{~L}^{-1}$ & 30 & 0,75 & IV \\
\hline 6 & Nicosulfuron & $\begin{array}{c}\text { Sanson } 40 \\
\text { CS }\end{array}$ & Sulfoniluréias & $\mathrm{CS}$ & $40 \mathrm{~g} \mathrm{~L}^{-1}$ & 60 & 1,5 & IV \\
\hline 7 & Nicosulfuron & $\begin{array}{c}\text { Sanson } 40 \\
\text { CS }\end{array}$ & Sulfoniluréias & $\mathrm{CS}$ & $40 \mathrm{~g} \mathrm{~L}^{-1}$ & 120 & 3,0 & IV \\
\hline
\end{tabular}

Fonte: ANDREI (2005) 
Para aplicação dos produtos utilizou-se um pulverizador costal à base de $\mathrm{CO}_{2}$, munido de barra com 6 bicos tipo leque 110-SF-02, espaçados de $0,50 \mathrm{~m}$, com pressão constante de 2,5 BAR e volume calda de $200 \mathrm{~L} \mathrm{ha}^{-1}$.

Os produtos foram aplicados quando o milho estava com 4 a 6 folhas e as condições climáticas eram: temperatura $24^{\circ} \mathrm{C}$, umidade relativa do ar $82 \%$ e velocidade do vento $2,1 \mathrm{~km} \mathrm{~h}^{-1}$.

As avaliações foram realizadas seguindo os parâmetros da Sociedade Brasileira da Ciência das Plantas Daninhas (SBCPD, 1995). Avaliaram-se os seguintes parâmetros: fitotoxicidade (de acordo com a Escala de Fitotoxicidade da E.W.R.C. - European Weed Research (Council), através de diagnose visual aos 7, 14, 28 e 42 dias após aplicação; altura de plantas aos 7, 14, 28 e 42 dias após a aplicação dos herbicidas, em dez plantas por parcela útil (nas duas linhas centrais), da base até o ápice; altura de inserção da espiga, aos 120 dias após a semeadura, avaliando-se dez plantas por parcela útil, onde cada planta foi medida da base até a inserção da primeira espiga; componentes de produção (massa de espigas, número de grãos por espiga e massa de 1000 grãos) e Produtividade (para a avaliação da produtividade, colheu-se as duas linhas centrais de cada parcela, com 5,0 metros de comprimento, corrigiu-se a umidade para $13 \%$ e os valores obtidos foram convertidos de gramas para $\mathrm{kg} \mathrm{ha}^{-1}$ ).

Os dados obtidos foram submetidos à análise de variância e as médias obtidas foram comparadas pelo teste de agrupamento de Skott-Knott, a 5\% de probabilidade.

\section{Resultados e discussão}

Os dados de fitotoxicidade dos herbicidas à cultura do milho, avaliados através da escala da E.W.R.C. estão descritos na Tabela 2. Observa-se que 7 dias após a aplicação dos herbicidas, houve uma pequena alteração foliar, caracterizada por descolorações e deformações (nota 2 na escala da E.W.R.C.). Essas deformações foram caracterizadas por um leve sintoma de enrugamento, principalmente para as maiores doses dos herbicidas e, principalmente no híbrido AS 1548, no qual esses sintomas apareceram em um maior número de plantas. Esses sintomas permaneceram até os 14 dias após a aplicação dos herbicidas, desaparecendo naturalmente após 21 dias, sendo que todos os híbridos se recuperaram de maneira semelhante.

Resultados semelhantes foram obtidos por DAMIÃO FILHO; MORO; TAVEIRA, (1996), GREEN; ULRICH (1994), PEREIRA FILHO; OLIVEIRA; PIRES (2000), MORO; DAMIÃO FILHO (1999); ZAGONEL (2003) e fisiologicamente explicados por DAMIÃO FILHO; MORO; TAVEIRA, (1996), GREEN; ULRICH (1994), GREEN (1998); MONKS; MULLINS; JOHSON (1992).

Os dados referentes à influência dos herbicidas nicosulfuron e foramsulfuron + iodosulfuron methyl sodium na altura de plantas dos híbridos de milho testados encontram-se na Tabela 2. Na avaliação realizada no momento da aplicação dos herbicidas, não houve diferença significativa entre os tratamentos, o que mostra que o milho apresentava um desenvolvimento uniforme quando da aplicação dos produtos. Portanto, quaisquer alterações na altura das plantas poderá ser creditada a um efeito dos herbicidas, tendo em vista que os demais fatores estavam sob controle. 
Tabela 2. Fitotoxicidade dos herbicidas à cultura do milho, avaliados pela escala da E.W.R.C. Marechal Cândido Rondon/PR, 2004/2005.

\begin{tabular}{|c|c|c|c|c|c|c|}
\hline \multirow{9}{*}{$\begin{array}{l}\frac{\infty}{0} \\
\stackrel{0}{0} \\
\frac{0}{4}\end{array}$} & \multirow{2}{*}{ Tratamentos } & \multirow{2}{*}{$\begin{array}{c}\text { Dose } \\
\left(\mathrm{g} \text { i.a. } \text { ha }^{-1}\right)\end{array}$} & \multicolumn{4}{|c|}{ Fitotoxicidade (E.W.R.C.) } \\
\hline & & & 7 DAA & 14 DAA & 28 DAA & 42 DAA \\
\hline & 1. Testemunha & & 1 & 1 & 1 & 1 \\
\hline & 2. Forams. + Iodos. & $22,5+1,5$ & 2 & 1 & 1 & 1 \\
\hline & 3. Forams. + Iodos. & $45,0+3,0$ & 2 & 2 & 1 & 1 \\
\hline & 4. Forams. + Iodos. & $90,0+6,0$ & 3 & 2 & 1 & 1 \\
\hline & 5. Nicosulfuron & 30,0 & 1 & 1 & 1 & 1 \\
\hline & 6. Nicosulfuron & 60,0 & 2 & 1 & 1 & 1 \\
\hline & 7. Nicosulfuron & 120,0 & 2 & 2 & 1 & 1 \\
\hline \multirow{9}{*}{$\begin{array}{l}\frac{10}{4} \\
\frac{10}{2}\end{array}$} & \multirow{2}{*}{ Tratamentos } & Dose & \multicolumn{4}{|c|}{ Fitotoxicidade (E.W.R.C.) } \\
\hline & & $\left(\right.$ g i.a. ha $\left.{ }^{-1}\right)$ & 7 DAA & 14 DAA & 28 DAA & 42 DAA \\
\hline & 1. Testemunha & - & 1 & 1 & 1 & 1 \\
\hline & 2. Forams. + Iodos. & $22,5+1,5$ & 1 & 1 & 1 & 1 \\
\hline & 3. Forams. + Iodos. & $45,0+3,0$ & 2 & 2 & 1 & 1 \\
\hline & 4. Forams. + Iodos. & $90,0+6,0$ & 2 & 2 & 1 & 1 \\
\hline & 5. Nicosulfuron & 30,0 & 2 & 1 & 1 & 1 \\
\hline & 6. Nicosulfuron & 60,0 & 2 & 1 & 1 & 1 \\
\hline & 7. Nicosulfuron & 120,0 & 2 & 2 & 1 & 1 \\
\hline \multirow{9}{*}{$\begin{array}{l}\stackrel{a}{\stackrel{1}{1}} \\
\text { N } \\
\text { ñ }\end{array}$} & \multirow{2}{*}{ Tratamentos } & Dose & \multicolumn{4}{|c|}{ Fitotoxicidade (E.W.R.C.) } \\
\hline & & (g i.a. ha- $\left.\mathbf{h}^{-1}\right)$ & 7 DAA & 14 DAA & 28 DAA & 42 DAA \\
\hline & 1. Testemunha & 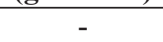 & 1 & 1 & 1 & 1 \\
\hline & 2. Forams. + Iodos. & $22,5+1,5$ & 1 & 1 & 1 & 1 \\
\hline & 3. Forams. + Iodos. & $45,0+3,0$ & 2 & 2 & 1 & 1 \\
\hline & 4. Forams. + Iodos. & $90,0+6,0$ & 2 & 2 & 1 & 1 \\
\hline & 5. Nicosulfuron & 30,0 & 2 & 1 & 1 & 1 \\
\hline & 6. Nicosulfuron & 60,0 & 2 & 1 & 1 & 1 \\
\hline & 7. Nicosulfuron & 120,0 & 2 & 2 & 1 & 1 \\
\hline \multirow{9}{*}{$\begin{array}{l}\text { m̃ } \\
\text { n }\end{array}$} & \multirow{2}{*}{ Tratamentos } & Dose & \multicolumn{4}{|c|}{ Fitotoxicidade (E.W.R.C.) } \\
\hline & & $\left(\mathrm{g}\right.$ i.a. ha $\left.^{-1}\right)$ & 7 DAA & 14 DAA & 28 DAA & 42 DAA \\
\hline & 1. Testemunha & & 1 & 1 & 1 & 1 \\
\hline & 2. Forams. + Iodos. & $22,5+1,5$ & 1 & 1 & 1 & 1 \\
\hline & 3. Forams. + Iodos. & $45,0+3,0$ & 2 & 1 & 1 & 1 \\
\hline & 4. Forams. + Iodos. & $90,0+6,0$ & 2 & 1 & 1 & 1 \\
\hline & 5. Nicosulfuron & 30,0 & 1 & 1 & 1 & 1 \\
\hline & 6. Nicosulfuron & 60,0 & 2 & 1 & 1 & 1 \\
\hline & 7. Nicosulfuron & 120,0 & 2 & 1 & 1 & 1 \\
\hline \multirow{9}{*}{ 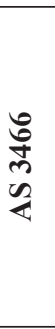 } & \multirow{2}{*}{ Tratamentos } & Dose & \multicolumn{4}{|c|}{ Fitotoxicidade (E.W.R.C.) } \\
\hline & & $\left(\mathrm{g}\right.$ i.a. $\left.\mathrm{ha}^{-1}\right)$ & 7 DAA & 14 DAA & 28 DAA & 42 DAA \\
\hline & 1. Testemunha & - & 1 & 1 & 1 & 1 \\
\hline & 2. Forams. + Iodos. & $22,5+1,5$ & 1 & 1 & 1 & 1 \\
\hline & 3. Forams. + Iodos. & $45,0+3,0$ & 2 & 2 & 1 & 1 \\
\hline & 4. Forams. + Iodos. & $90,0+6,0$ & 2 & 2 & 1 & 1 \\
\hline & 5. Nicosulfuron & 30,0 & 2 & 1 & 1 & 1 \\
\hline & 6. Nicosulfuron & 60,0 & 2 & 2 & 1 & 1 \\
\hline & 7. Nicosulfuron & 120,0 & 2 & 2 & 1 & 1 \\
\hline \multirow{9}{*}{$\begin{array}{l}\frac{m}{n} \\
\text { n } \\
\text { n }\end{array}$} & \multirow{2}{*}{ Tratamentos } & Dose & \multicolumn{4}{|c|}{ Fitotoxicidade (E.W.R.C.) } \\
\hline & & $\left.(\text { g i.a. ha })^{-1}\right)$ & 7 DAA & 14 DAA & 28 DAA & 42 DAA \\
\hline & 1. Testemunha & & 1 & 1 & 1 & 1 \\
\hline & 2. Forams. + Iodos. & $22,5+1,5$ & 1 & 1 & 1 & 1 \\
\hline & 3. Forams. + Iodos. & $45,0+3,0$ & 2 & 1 & 1 & 1 \\
\hline & 4. Forams. + Iodos. & $90,0+6,0$ & 2 & 2 & 1 & 1 \\
\hline & 5. Nicosulfuron & 30,0 & 1 & 1 & 1 & 1 \\
\hline & 6. Nicosulfuron & 60,0 & 2 & 1 & 1 & 1 \\
\hline & 7. Nicosulfuron & 120,0 & 2 & 2 & 1 & 1 \\
\hline
\end{tabular}

Fonte: European Weed Research Council (1964). 
Para o híbrido AS 1545 na avaliação realizada 7 dias após a aplicação, pode-se observar que houve interferência significativa na altura das plantas, em função da aplicação dos herbicidas, sendo que todas as doses utilizadas influenciaram negativamente $o$ crescimento das plantas, quando comparadas com a testemunha.

Para esse híbrido, o herbicida foramsulfuron + iodosulfuron methyl sodium mostrou-se mais fitotóxico, quando comparado com o herbicida nicosulfuron.

Para o híbrido AS 1548, observa-se uma menor influência dos herbicidas na altura de plantas, sendo que o herbicida foramsulfuron + iodosulfuron methyl sodium apresentou influência significativa apenas aos 7 dias após a aplicação, quando comparado com a testemunha. A partir dos 14 dias após a aplicação, não houve mais influência dos herbicidas, tendo as plantas se recuperado, retomando seu crescimento normal.

Analisando-se os dados da Tabela 3, observa-se que, para o híbrido AS 3466 não houve interferência dos herbicidas na altura das plantas, mostrando, portanto, menor sensibilidade desse híbrido aos herbicidas, mesmo nas maiores doses. O híbrido AS 1533 também apresentou menor sensibilidade aos herbicidas, pois não houve diferença significativa entre os tratamentos. Para esse parâmetro, os herbicidas foramsulfuron + iodosulfuron methyl sodium e nicosulfuron não afetaram as plantas de milho.

O herbicida foramsulfuron + iodosulfuron methyl sodium ocasionou uma redução no crescimento das plantas de milho, cultivar AS 32, sendo que essa redução ocorreu na avaliação realizada aos 7 (nas duas maiores doses) e aos 14 dias após a aplicação (na maior dose). A partir dos 21 dias após a aplicação, os herbicidas já não influenciaram significativamente o crescimento das plantas.
Observa-se que, para o híbrido S2 709, o herbicida foramsulfuron + iodosulfuron methyl sodium apresentou redução no crescimento das plantas, apenas aos 7 dias após a aplicação, para as duas maiores doses utilizadas no experimento (Tabela 3 ). Já para o herbicida nicosulfuron, essa redução só foi estatisticamente significativa, na maior dose. Para todos os produtos, em todas as doses, a partir dos 14 dias após a aplicação, as plantas recuperaram seu desenvolvimento.

Analisando-se conjuntamente os dados da Tabela 3 , pode-se observar que, apesar de alguns híbridos apresentarem maior sensibilidade aos herbicidas, essa sensibilidade não influenciou na altura das plantas, pois as mesmas apresentaram, ao final das avaliações, crescimento igual ao da testemunha, o que vem de encontro aos resultados obtidos nos experimentos conduzidos por (PEREIRA FILHO; OLIVEIRA; PIRES, 2000; ZAGONEL, 2003), mas contradiz os obtidos por(DAMIÃO FILHO; MORO; TAVEIRA,. 1996), que encontraram interação significativa na altura média das plantas entre os híbridos estudados e a aplicação de nicosulfuron.

Para a influência dos herbicidas nicosulfuron e foramsulfuron + iodosulfuron methyl sodium na altura de inserção da espiga nos híbridos de milho (Tabela 4), observa-se que o híbrido AS 1533 foi o mais sensível aos herbicidas, pois apenas a menor dose do herbicida foramsulfuron + iodosulfuron methyl sodium não apresentou diferença estatística em relação à testemunha. O híbrido S2 709 foi influenciado pelas duas maiores doses do herbicida foramsulfuron + iodosulfuron methyl sodium, e não sofreu nenhuma influência do herbicida nicosulfuron. Já para o híbrido AS 1545 observase redução na altura de inserção das espigas apenas nas duas maiores doses, para ambos os produtos. O híbrido AS 3466 teve altura reduzida nas doses maiores de cada herbicida utilizado. Para os demais híbridos, não houve influência dos herbicidas. 
Tabela 3. Altura das plantas de milho em função dos herbicidas nicosulfuron e foramsulfuron + iodosulfuron methyl sodium. Marechal Cândido Rondon/PR, 2004/2005.

\begin{tabular}{|c|c|c|c|c|c|c|c|}
\hline \multirow{11}{*}{ 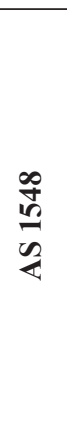 } & \multirow{2}{*}{ Tratamentos } & \multirow{2}{*}{$\begin{array}{c}\text { Dose } \\
\left(\text { g i.a. } \text { ha }^{-1}\right) \\
\end{array}$} & \multicolumn{5}{|c|}{ Altura de Plantas (cm) } \\
\hline & & & Pré-Aval. & 7 DAA & 14 DAA & 28 DAA & 42 DAA \\
\hline & 1. Testemunha & - & $37,66 \mathrm{a}^{*}$ & $51,80 \mathrm{a}$ & $88,25 \mathrm{a}$ & $144,53 \mathrm{a}$ & $198,55 \mathrm{a}$ \\
\hline & 2. Forams. + Iodos. & $22,5+1,5$ & $37,34 \mathrm{a}$ & $48,58 \mathrm{~b}$ & $84,00 \mathrm{a}$ & $144,20 \mathrm{a}$ & $197,75 \mathrm{a}$ \\
\hline & 3. Forams. + Iodos. & $45,0+3,0$ & $37,52 \mathrm{a}$ & $50,73 \mathrm{a}$ & $89,70 \mathrm{a}$ & $147,70 \mathrm{a}$ & $199,98 \mathrm{a}$ \\
\hline & 4. Forams. + Iodos. & $90,0+6,0$ & $37,60 \mathrm{a}$ & $47,60 \mathrm{~b}$ & $83,03 \mathrm{a}$ & $139,28 \mathrm{a}$ & $195,23 \mathrm{a}$ \\
\hline & 5. Nicosulfuron & 30,0 & $37,82 \mathrm{a}$ & $53,58 \mathrm{a}$ & $91,23 \mathrm{a}$ & $151,00 \mathrm{a}$ & $201,63 \mathrm{a}$ \\
\hline & 6. Nicosulfuron & 60,0 & $37,52 \mathrm{a}$ & $53,93 \mathrm{a}$ & $91,13 \mathrm{a}$ & $150,10 \mathrm{a}$ & $198,80 \mathrm{a}$ \\
\hline & 7. Nicosulfuron & 120,0 & $37,08 \mathrm{a}$ & $51,48 \mathrm{a}$ & $88,33 \mathrm{a}$ & $149,55 \mathrm{a}$ & $199,98 \mathrm{a}$ \\
\hline & $\mathrm{F}$ & - & $0,458^{\mathrm{NS}}$ & $2,913^{*}$ & $1,899^{\mathrm{NS}}$ & $2,243^{\mathrm{NS}}$ & $0,738^{\mathrm{NS}}$ \\
\hline & C.V. $(\%)$ & - & 2,09 & 6,05 & 6,02 & 4,26 & 2,64 \\
\hline \multirow{10}{*}{$\frac{n}{\frac{10}{2}} \frac{1}{\infty}$} & Tratamentos & $\begin{array}{c}\text { Dose } \\
\left(\mathrm{g} \text { i.a. } \text { ha }^{-1}\right)\end{array}$ & Pré-Aval. & 7 DAA & 14 DAA & 28 DAA & 42 DAA \\
\hline & 1. Testemunha & - & $35,14 \mathrm{a}$ & $55,85 \mathrm{a}$ & $86,98 \mathrm{a}$ & $163,68 \mathrm{a}$ & $217,05 \mathrm{a}$ \\
\hline & 2. Forams. + Iodos. & $22,5+1,5$ & $34,46 \mathrm{a}$ & $46,03 \mathrm{~d}$ & $83,00 \mathrm{~b}$ & $152,28 \mathrm{~b}$ & $214,08 \mathrm{a}$ \\
\hline & 3. Forams. + Iodos. & $45,0+3,0$ & $34,88 \mathrm{a}$ & $46,83 \mathrm{~d}$ & $87,23 \mathrm{~b}$ & $146,43 \mathrm{~b}$ & $218,35 \mathrm{a}$ \\
\hline & 4. Forams. + Iodos. & $90,0+6,0$ & $34,62 \mathrm{a}$ & $45,50 \mathrm{~d}$ & $84,95 \mathrm{~b}$ & $149,18 \mathrm{~b}$ & $218,30 \mathrm{a}$ \\
\hline & 5. Nicosulfuron & 30,0 & $34,43 \mathrm{a}$ & $51,63 \mathrm{~b}$ & $90,35 \mathrm{a}$ & $150,00 \mathrm{~b}$ & $219,15 \mathrm{a}$ \\
\hline & 6. Nicosulfuron & 60,0 & $35,33 \mathrm{a}$ & $51,75 \mathrm{~b}$ & $83,00 \mathrm{~b}$ & $150,33 \mathrm{~b}$ & $217,53 \mathrm{a}$ \\
\hline & 7. Nicosulfuron & 120,0 & $34,60 \mathrm{a}$ & $49,43 \mathrm{c}$ & $87,05 \mathrm{a}$ & $154,60 \mathrm{~b}$ & $218,36 \mathrm{a}$ \\
\hline & $\mathrm{F}$ & - & $2,151^{\mathrm{NS}}$ & $17,905^{*}$ & $2,841^{*}$ & $5,271^{*}$ & $0,466^{\mathrm{NS}}$ \\
\hline & C.V. $(\%)$ & - & 1,52 & 4,03 & 4,05 & 3,58 & 2,51 \\
\hline \multirow{10}{*}{ 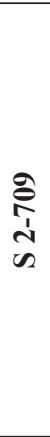 } & Tratamentos & $\begin{array}{c}\text { Dose } \\
\left(\text { g i.a. } \text { ha }^{-1}\right) \\
\end{array}$ & Pré-Aval. & 7 DAA & 14 DAA & 28 DAA & 42 DAA \\
\hline & 1. Testemunha & - & $28,70 \mathrm{a}$ & $45,68 \mathrm{a}$ & $78,20 \mathrm{a}$ & $131,83 \mathrm{a}$ & $204,78 \mathrm{a}$ \\
\hline & 2. Forams. + Iodos. & $22,5+1,5$ & $28,48 \mathrm{a}$ & $43,08 \mathrm{a}$ & $77,65 \mathrm{a}$ & $128,28 \mathrm{a}$ & $199,18 \mathrm{a}$ \\
\hline & 3. Forams. + Iodos. & $45,0+3,0$ & $28,35 \mathrm{a}$ & $40,10 \mathrm{~b}$ & $70,88 \mathrm{a}$ & $124,63 \mathrm{a}$ & $188,93 \mathrm{a}$ \\
\hline & 4. Forams. + Iodos. & $90,0+6,0$ & $28,78 \mathrm{a}$ & $39,33 \mathrm{~b}$ & $76,83 \mathrm{a}$ & $127,20 \mathrm{a}$ & $197,50 \mathrm{a}$ \\
\hline & 5. Nicosulfuron & 30,0 & $28,05 \mathrm{a}$ & $43,93 \mathrm{a}$ & 83,33 a & $130,85 \mathrm{a}$ & $199,18 \mathrm{a}$ \\
\hline & 6. Nicosulfuron & 60,0 & $28,10 \mathrm{a}$ & $42,85 \mathrm{a}$ & $82,33 \mathrm{a}$ & $131,78 \mathrm{a}$ & $199,73 \mathrm{a}$ \\
\hline & 7. Nicosulfuron & 120,0 & $28,30 \mathrm{a}$ & $39,43 \mathrm{~b}$ & $76,18 \mathrm{a}$ & $126,75 \mathrm{a}$ & $195,93 \mathrm{a}$ \\
\hline & $\mathrm{F}$ & - & $0,294^{\mathrm{NS}}$ & $3,270^{*}$ & $2,789^{*}$ & $0,419^{\mathrm{NS}}$ & $1,578^{\mathrm{NS}}$ \\
\hline & C.V. $(\%)$ & - & 4,04 & 7,24 & 7,10 & 7,48 & 4,32 \\
\hline \multirow{10}{*}{ लै } & Tratamentos & $\begin{array}{c}\text { Dose } \\
\left(\mathrm{g} \text { i.a. } \text { ha }^{-1}\right)\end{array}$ & Pré-Aval. & 7 DAA & 14 DAA & 28 DAA & 42 DAA \\
\hline & 1. Testemunha & - & $25,98 \mathrm{a}$ & $47,45 \mathrm{a}$ & $83,65 \mathrm{a}$ & $139,40 \mathrm{a}$ & $184,15 \mathrm{a}$ \\
\hline & 2. Forams. + Iodos. & $22,5+1,5$ & $26,95 \mathrm{a}$ & $45,08 \mathrm{a}$ & $85,80 \mathrm{a}$ & $141,93 \mathrm{a}$ & $202,60 \mathrm{a}$ \\
\hline & 3. Forams. + Iodos. & $45,0+3,0$ & $27,18 \mathrm{a}$ & $43,80 \mathrm{~b}$ & $83,03 \mathrm{a}$ & $142,93 \mathrm{a}$ & $201,78 \mathrm{a}$ \\
\hline & 4. Forams. + Iodos. & $90,0+6,0$ & $26,65 \mathrm{a}$ & $41,28 \mathrm{~b}$ & $77,78 \mathrm{~b}$ & $140,80 \mathrm{a}$ & $200,18 \mathrm{a}$ \\
\hline & 5. Nicosulfuron & 30,0 & $26,40 \mathrm{a}$ & $47,45 \mathrm{a}$ & $82,40 \mathrm{a}$ & $144,85 \mathrm{a}$ & $204,10 \mathrm{a}$ \\
\hline & 6. Nicosulfuron & 60,0 & $26,18 \mathrm{a}$ & $45,95 \mathrm{a}$ & $80,68 \mathrm{~b}$ & $139,70 \mathrm{a}$ & $202,23 \mathrm{a}$ \\
\hline & 7. Nicosulfuron & 120,0 & $26,18 \mathrm{a}$ & $45,98 \mathrm{a}$ & $84,60 \mathrm{a}$ & $142,83 \mathrm{a}$ & $201,38 \mathrm{a}$ \\
\hline & $\mathrm{F}$ & - & $0,539^{\mathrm{NS}}$ & $5,848^{*}$ & $3,488^{*}$ & $0,645^{\mathrm{NS}}$ & $0,763^{\mathrm{NS}}$ \\
\hline & C.V. $(\%)$ & - & 5,10 & 4,45 & 3,86 & 3,83 & 8,70 \\
\hline \multirow{10}{*}{ 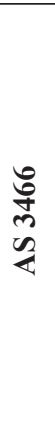 } & Tratamentos & $\begin{array}{c}\text { Dose } \\
\left(\text { g i.a. } \text { ha }^{-1}\right)\end{array}$ & Pré-Aval. & 7 DAA & 14 DAA & 28 DAA & 42 DAA \\
\hline & 1. Testemunha & - & $29,60 \mathrm{a}$ & $47,85 \mathrm{a}$ & $87,95 \mathrm{a}$ & $141,43 \mathrm{a}$ & $201,58 \mathrm{a}$ \\
\hline & 2. Forams. + Iodos. & $22,5+1,5$ & $29,58 \mathrm{a}$ & $47,83 \mathrm{a}$ & $85,10 \mathrm{a}$ & $142,98 \mathrm{a}$ & $197,88 \mathrm{a}$ \\
\hline & 3. Forams. + Iodos. & $45,0+3,0$ & $29,38 \mathrm{a}$ & $47,90 \mathrm{a}$ & $83,53 \mathrm{a}$ & $138,05 \mathrm{a}$ & $200,28 \mathrm{a}$ \\
\hline & 4. Forams. + Iodos. & $90,0+6,0$ & $28,72 \mathrm{a}$ & $46,58 \mathrm{a}$ & $81,65 \mathrm{a}$ & $138,68 \mathrm{a}$ & $196,48 \mathrm{a}$ \\
\hline & 5. Nicosulfuron & 30,0 & $29,78 \mathrm{a}$ & $48,90 \mathrm{a}$ & $85,13 \mathrm{a}$ & $141,75 \mathrm{a}$ & $198,10 \mathrm{a}$ \\
\hline & 6. Nicosulfuron & 60,0 & $30,08 \mathrm{a}$ & $49,00 \mathrm{a}$ & $83,53 \mathrm{a}$ & $139,45 \mathrm{a}$ & $199,73 \mathrm{a}$ \\
\hline & 7. Nicosulfuron & 120,0 & $29,55 \mathrm{a}$ & $49,43 \mathrm{a}$ & $83,75 \mathrm{a}$ & $139,75 \mathrm{a}$ & $198,13 \mathrm{a}$ \\
\hline & $\mathrm{F}$ & - & $0,797^{\mathrm{NS}}$ & $0,973^{\mathrm{NS}}$ & $1,109^{\mathrm{NS}}$ & $0,620^{\mathrm{NS}}$ & $1,290^{\mathrm{NS}}$ \\
\hline & C.V. $(\%)$ & - & 3,54 & 4,55 & 4,94 & 3,62 & 1,71 \\
\hline
\end{tabular}


Contiero, R. L.

\begin{tabular}{|c|c|c|c|c|c|c|c|}
\hline \multirow{10}{*}{$\frac{m}{n}$} & Tratamentos & $\begin{array}{c}\text { Dose } \\
\left(\mathrm{g} \text { i.a. } \text { ha }^{-1}\right)\end{array}$ & Pré-Aval. & 7 DAA & 14 DAA & 28 DAA & 42 DAA \\
\hline & 1. Testemunha & - & $29,08 \mathrm{a}$ & $49,88 \mathrm{a}$ & $86,25 \mathrm{a}$ & $136,58 \mathrm{a}$ & $199,38 \mathrm{a}$ \\
\hline & 2. Forams. + Iodos. & $22,5+1,5$ & $28,90 \mathrm{a}$ & $46,85 \mathrm{a}$ & $80,95 \mathrm{a}$ & $130,05 \mathrm{a}$ & $199,13 \mathrm{a}$ \\
\hline & 3. Forams. + Iodos. & $45,0+3,0$ & $29,24 \mathrm{a}$ & $46,58 \mathrm{a}$ & $84,45 \mathrm{a}$ & 129,90 a & $194,65 \mathrm{a}$ \\
\hline & 4. Forams. + Iodos. & $90,0+6,0$ & $29,63 \mathrm{a}$ & $46,80 \mathrm{a}$ & $80,88 \mathrm{a}$ & $133,80 \mathrm{a}$ & $197,38 \mathrm{a}$ \\
\hline & 5. Nicosulfuron & 30,0 & $29,20 \mathrm{a}$ & $49,40 \mathrm{a}$ & $83,78 \mathrm{a}$ & $136,15 \mathrm{a}$ & $199,43 \mathrm{a}$ \\
\hline & 6. Nicosulfuron & 60,0 & $28,94 \mathrm{a}$ & $47,55 \mathrm{a}$ & $84,70 \mathrm{a}$ & $135,08 \mathrm{a}$ & $198,00 \mathrm{a}$ \\
\hline & 7. Nicosulfuron & 120,0 & $29,36 \mathrm{a}$ & $48,35 \mathrm{a}$ & $86,63 \mathrm{a}$ & $140,45 \mathrm{a}$ & $196,18 \mathrm{a}$ \\
\hline & $\mathrm{F}$ & - & $0,684^{\mathrm{NS}}$ & $2,029^{\mathrm{NS}}$ & $1,563^{\mathrm{NS}}$ & $1,904^{\mathrm{NS}}$ & $1,502^{\mathrm{NS}}$ \\
\hline & C.V. $(\%)$ & - & 2,33 & 4,34 & 4,51 & 4,41 & 1,66 \\
\hline
\end{tabular}

Fonte: *Médias seguidas de mesma letra, na coluna, não diferem estatisticamente entre si, pelo teste de Skott-Knott.

Tabela 4. Altura de inserção das espigas de milho em função dos herbicidas nicosulfuron e foramsulfuron + iodosulfuron methyl sodium. Marechal Cândido Rondon/PR, 2004/2005.

\begin{tabular}{|c|c|c|c|c|c|c|c|}
\hline \multirow{2}{*}{ Tratamentos } & \multirow{2}{*}{$\begin{array}{c}\text { Dose } \\
\left(\mathrm{g} \text { i.a. } \mathbf{h a}^{-1}\right)\end{array}$} & \multicolumn{6}{|c|}{ Altura de Inserção das Espigas (cm) } \\
\hline & & AS 32 & S2 709 & AS 1533 & AS 1545 & AS 1548 & AS 3466 \\
\hline 1. Testemunha & - & $120,53 \mathrm{a}^{*}$ & $101,68 \mathrm{a}$ & $119,18 \mathrm{a}$ & $109,50 \mathrm{a}$ & $98,63 \mathrm{a}$ & $120,93 \mathrm{a}$ \\
\hline 2. Forams. + Iodos. & $22,5+1,5$ & $120,26 \mathrm{a}$ & $98,60 \mathrm{a}$ & $117,00 \mathrm{a}$ & $108,03 \mathrm{a}$ & $100,25 \mathrm{a}$ & $120,90 \mathrm{a}$ \\
\hline 3. Forams. + Iodos. & $45,0+3,0$ & $120,63 \mathrm{a}$ & $98,70 \mathrm{~b}$ & $114,50 \mathrm{~b}$ & $110,05 \mathrm{a}$ & $100,08 \mathrm{a}$ & $117,73 \mathrm{~b}$ \\
\hline 4. Forams. + Iodos. & $90,0+6,0$ & $117,45 \mathrm{a}$ & $95,93 \mathrm{~b}$ & $115,25 \mathrm{~b}$ & $106,53 \mathrm{~b}$ & 98,38 a & $114,83 \mathrm{~b}$ \\
\hline 5. Nicosulfuron & 30,0 & $121,68 \mathrm{a}$ & 98,03 a & $113,68 \mathrm{~b}$ & $109,65 \mathrm{a}$ & $103,65 \mathrm{a}$ & $121,08 \mathrm{a}$ \\
\hline 6. Nicosulfuron & 60,0 & $119,53 \mathrm{a}$ & $98,50 \mathrm{a}$ & $114,35 \mathrm{~b}$ & $111,83 \mathrm{a}$ & $105,28 \mathrm{a}$ & $116,40 \mathrm{~b}$ \\
\hline 7. Nicosulfuron & 120,0 & $118,18 \mathrm{a}$ & $98,00 \mathrm{a}$ & $114,23 \mathrm{~b}$ & $103,80 \mathrm{~b}$ & $102,85 \mathrm{a}$ & $115,70 \mathrm{~b}$ \\
\hline $\mathrm{F}$ & - & $1,053^{\mathrm{NS}}$ & $0,993^{\mathrm{NS}}$ & $3,128^{*}$ & $4,016^{*}$ & $2,683^{\mathrm{NS}}$ & $11,243^{*}$ \\
\hline C.V. $(\%)$ & - & 2,695 & 3,862 & 2,118 & 2,699 & 3,567 & 1,259 \\
\hline
\end{tabular}

Fonte: *Médias seguidas de mesma letra, na coluna, não diferem estatisticamente entre si, pelo teste de Skott-Knott.

Resultados semelhantes aos obtidos no presente trabalho foram obtidos por diversos autores, que destacam que a sensibilidade da cultura do milho aos herbicidas (principalmente aos herbicidas testados) depende da dose utilizada e do híbrido testado, uma vez que existe comprovadamente uma tolerância diferencial de cultivares de milho aos herbicidas do grupo das sulfoniluréias (DAMIÃO FILHO; MORO; TAVEIRA, 1996; GREEN; ULRICH, 1994; MONKS; MULLINS. JOHSON, 1992; GREEN, 1998; FAHL; CARELLI, 1997;
LOPES-OVEJERO; FANCELLI; DOURADONETO, 2003; ZAGONEL, 2003).

Para a influência dos herbicidas nicosulfuron e foramsulfuron + iodosulfuron methyl sodium na massa de espigas de milho (Tabela 5) os dados mostram influência do herbicida foramsulfuron + iodosulfuron methyl sodium apenas no híbrido S2 709. Para os demais híbridos, não se observou influência dos herbicidas na massa das espigas, da mesma forma que (PEREIRA FILHO; OLIVEIRA; PIRES, 2000). 
Tabela 5. Efeito dos herbicidas nicosulfuron e foramsulfuron + iodosulfuron methyl sodium na massa das espigas de milho. Marechal Cândido Rondon/PR, 2004/2005.

\begin{tabular}{lccccccc}
\hline \multirow{2}{*}{ Tratamentos } & \multirow{2}{*}{$\begin{array}{c}\text { Dose } \\
\text { ig i.a. ha }\end{array}$} & \multicolumn{7}{c}{ Massa de Espigas (g) } \\
\cline { 3 - 7 } & - & AS 32 & S2 709 & AS 1533 & AS 1545 & AS 1548 & AS 3466 \\
\hline 1. Testemunha & - & $106,27 \mathrm{a}^{*}$ & $143,24 \mathrm{a}$ & $123,69 \mathrm{a}$ & $148,96 \mathrm{a}$ & $111,38 \mathrm{a}$ & $136,04 \mathrm{a}$ \\
2. Forams. + Iodos. & $22,5+1,5$ & $100,24 \mathrm{a}$ & $128,53 \mathrm{~b}$ & $126,29 \mathrm{a}$ & $143,73 \mathrm{a}$ & $108,86 \mathrm{a}$ & $127,48 \mathrm{a}$ \\
3. Forams. + Iodos. & $45,0+3,0$ & $104,44 \mathrm{a}$ & $137,46 \mathrm{a}$ & $122,86 \mathrm{a}$ & $152,32 \mathrm{a}$ & $114,63 \mathrm{a}$ & $124,43 \mathrm{a}$ \\
4. Forams. + Iodos. & $90,0+6,0$ & $107,22 \mathrm{a}$ & $117,57 \mathrm{~b}$ & $114,76 \mathrm{a}$ & $150,09 \mathrm{a}$ & $105,53 \mathrm{a}$ & $139,40 \mathrm{a}$ \\
5. Nicosulfuron & 30,0 & $104,68 \mathrm{a}$ & $131,93 \mathrm{a}$ & $119,65 \mathrm{a}$ & $147,37 \mathrm{a}$ & $122,51 \mathrm{a}$ & $132,63 \mathrm{a}$ \\
6. Nicosulfuron & 60,0 & $104,87 \mathrm{a}$ & $140,97 \mathrm{a}$ & $126,18 \mathrm{a}$ & $151,62 \mathrm{a}$ & $106,05 \mathrm{a}$ & $135,90 \mathrm{a}$ \\
7. Nicosulfuron & 120,0 & $104,28 \mathrm{a}$ & $135,55 \mathrm{a}$ & $121,16 \mathrm{a}$ & $146,79 \mathrm{a}$ & $112,65 \mathrm{a}$ & $125,37 \mathrm{a}$ \\
F & - & $0,197^{\mathrm{NS}}$ & $3,197^{*}$ & $0,482^{\mathrm{NS}}$ & $0,248^{\mathrm{NS}}$ & $1,539^{\mathrm{NS}}$ & $1,803^{\mathrm{NS}}$ \\
C.V. (\%) & - & 10,578 & 8,118 & 10,666 & 9,039 & 9,423 & 7,439 \\
\hline
\end{tabular}

Fonte: *Médias seguidas de mesma letra, na coluna, não diferem estatisticamente entre si, pelo teste de Skott-Knott.

Os dados referentes à influência dos herbicidas nicosulfuron e foramsulfuron + iodosulfuron methyl sodium no número de grãos por espiga de milho dos híbridos testados encontram-se na Tabela 6. Os híbridos mais sensíveis ao herbicida nicosulfuron foram o AS 32 (nas duas maiores doses) e o AS 1533 (em todas as doses testadas), o que concorda com os dados obtidos por (LÓPEZ-OVEJERO; FANCELLI; DOURADO-NETO, 2003). Para os demais híbridos, não houve influência dos herbicidas.

Tabela 6. Número de grãos por espigas de milho em função dos herbicidas nicosulfuron e foramsulfuron + iodosulfuron methyl sodium. Marechal Cândido Rondon/PR, 2004/2005.

\begin{tabular}{lccccccc}
\hline \multirow{2}{*}{ Tratamentos } & \multirow{2}{*}{$\begin{array}{c}\text { Dose } \\
\text { i.a. ha }\end{array}$} & \multicolumn{7}{c}{ Número de Grãos por Espiga } \\
\cline { 3 - 7 } & - & $\mathbf{A S ~ 3 2}$ & $\mathbf{S 2 ~ 7 0 9}$ & $\mathbf{A S ~ 1 5 3 3}$ & $\mathbf{A S ~ 1 5 4 5}$ & $\mathbf{A S ~ 1 5 4 8}$ & $\mathbf{A S ~ 3 4 6 6}$ \\
\hline 1. Testemunha & - & $421,45 \mathrm{~b}^{*}$ & $626,67 \mathrm{a}$ & $363,66 \mathrm{a}$ & $514,34 \mathrm{a}$ & $450,02 \mathrm{a}$ & $470,14 \mathrm{a}$ \\
2. Forams. + Iodos. & $22,5+1,5$ & $467,26 \mathrm{a}$ & $631,30 \mathrm{a}$ & $390,52 \mathrm{a}$ & $537,19 \mathrm{a}$ & $483,59 \mathrm{a}$ & $413,75 \mathrm{a}$ \\
3. Forams. + Iodos. & $45,0+3,0$ & $494,22 \mathrm{a}$ & $586,89 \mathrm{a}$ & $383,07 \mathrm{a}$ & $526,57 \mathrm{a}$ & $445,20 \mathrm{a}$ & $429,57 \mathrm{a}$ \\
4. Forams. + Iodos. & $90,0+6,0$ & $468,07 \mathrm{a}$ & $586,35 \mathrm{a}$ & $329,05 \mathrm{a}$ & $515,55 \mathrm{a}$ & $467,05 \mathrm{a}$ & $425,98 \mathrm{a}$ \\
5. Nicosulfuron & 30,0 & $474,00 \mathrm{a}$ & $588,35 \mathrm{a}$ & $334,80 \mathrm{~b}$ & $556,43 \mathrm{a}$ & $488,96 \mathrm{a}$ & $414,34 \mathrm{a}$ \\
6. Nicosulfuron & 60,0 & $425,45 \mathrm{~b}$ & $621,79 \mathrm{a}$ & $348,08 \mathrm{~b}$ & $557,47 \mathrm{a}$ & $439,98 \mathrm{a}$ & $398,81 \mathrm{a}$ \\
7. Nicosulfuron & 120,0 & $439,52 \mathrm{~b}$ & $593,53 \mathrm{a}$ & $376,92 \mathrm{~b}$ & $510,81 \mathrm{a}$ & $442,98 \mathrm{a}$ & $415,79 \mathrm{a}$ \\
F & - & $3,387^{*}$ & $0,797^{\mathrm{NS}}$ & $3,349^{*}$ & $1,021^{\mathrm{NS}}$ & $1,780^{\mathrm{NS}}$ & $1,669^{\mathrm{NS}}$ \\
C.V. $(\%)$ & - & 7,266 & 8,497 & 8,170 & 8,208 & 7,372 & 9,224 \\
\hline
\end{tabular}

Fonte: *Médias seguidas de mesma letra, na coluna, não diferem estatisticamente entre si, pelo teste de Skott-Knott.

Quanto à influência dos herbicidas nicosulfuron e foramsulfuron + iodosulfuron methyl sodium na massa de 1000 grãos (Tabela 7), observa-se que só houve influência significativa para o híbrido
AS 1548. No caso do herbicida foramsulfuron + iodosulfuron methyl sodium, a interação aconteceu nas duas maiores doses, e no caso do herbicida nicosulfuron, na maior dose. 
Tabela 7. Massa de 1000 grãos de milho em função dos herbicidas nicosulfuron e foramsulfuron + iodosulfuron methyl sodium. Marechal Cândido Rondon/PR, 2004/2005.

\begin{tabular}{lccccccc}
\hline \multirow{2}{*}{ Tratamentos } & \multirow{2}{*}{$\begin{array}{c}\text { Dose } \\
\text { i.a. ha-1) }\end{array}$} & \multicolumn{7}{c}{ Massa de 1000 Grãos (g) } \\
\cline { 3 - 7 } & - & AS 32 & S2 709 & AS 1533 & AS 1545 & AS 1548 & AS 3466 \\
\hline 1. Testemunha & - & $184,30 \mathrm{a}^{*}$ & $190,42 \mathrm{a}$ & $273,32 \mathrm{a}$ & $208,22 \mathrm{a}$ & $184,15 \mathrm{a}$ & $231,23 \mathrm{a}$ \\
2. Forams. + Iodos. & $22,5+1,5$ & $172,13 \mathrm{a}$ & $167,61 \mathrm{a}$ & $252,48 \mathrm{a}$ & $211,38 \mathrm{a}$ & $194,59 \mathrm{a}$ & $247,57 \mathrm{a}$ \\
3. Forams. + Iodos. & $45,0+3,0$ & $177,97 \mathrm{a}$ & $195,44 \mathrm{a}$ & $257,96 \mathrm{a}$ & $227,06 \mathrm{a}$ & $170,72 \mathrm{~b}$ & $226,74 \mathrm{a}$ \\
4. Forams. + Iodos. & $90,0+6,0$ & $183,20 \mathrm{a}$ & $162,33 \mathrm{a}$ & $256,30 \mathrm{a}$ & $216,44 \mathrm{a}$ & $172,51 \mathrm{~b}$ & $258,43 \mathrm{a}$ \\
5. Nicosulfuron & 30,0 & $180,57 \mathrm{a}$ & $185,02 \mathrm{a}$ & $268,71 \mathrm{a}$ & $225,05 \mathrm{a}$ & $191,68 \mathrm{a}$ & $253,80 \mathrm{a}$ \\
6. Nicosulfuron & 60,0 & $197,75 \mathrm{a}$ & $187,99 \mathrm{a}$ & $276,12 \mathrm{a}$ & $218,65 \mathrm{a}$ & $187,30 \mathrm{a}$ & $270,26 \mathrm{a}$ \\
7. Nicosulfuron & 120,0 & $198,77 \mathrm{a}$ & $184,63 \mathrm{a}$ & $259,37 \mathrm{a}$ & $224,29 \mathrm{a}$ & $174,77 \mathrm{~b}$ & $243,31 \mathrm{a}$ \\
F & - & $2,825^{\text {NS }}$ & $1,731^{\text {NS }}$ & $0,889^{\text {NS }}$ & $0,541^{\text {NS }}$ & $4,161^{*}$ & $2,155^{\text {NS }}$ \\
C.V. (\%) & - & 7,137 & 11,426 & 8,252 & 9,996 & 5,780 & 8,384 \\
\hline
\end{tabular}

Fonte: *Médias seguidas de mesma letra, na coluna, não diferem estatisticamente entre si, pelo teste de Skott-Knott.

Para a influência dos herbicidas nicosulfuron e foramsulfuron + iodosulfuron methyl sodium na produtividade dos híbridos de milho (Tabela 8), vale salientar que o experimento apresentou baixa produtividade (média de $2.560 \mathrm{~kg} \mathrm{ha}^{-1}$ ), principalmente em função da baixa precipitação pluviométrica ocorrida no período $(576,58 \mathrm{~mm}$, de acordo com os dados obtidos na estação meteorológica instalada na Estação Experimental da UNIOESTE, local de instalação do ensaio). Segundo a Embrapa Milho e Sorgo (2008), as maiores produtividades têm ocorrido associadas a consumos de água entre 500 e $800 \mathrm{~mm}$ considerando todo o ciclo da cultura, sendo que no período que vai da iniciação floral à maturação (planta em torno de $30 \mathrm{~cm}$ de altura), o consumo pode atingir 5 a 7 $\mathrm{mm} /$ dia. Dessa forma, observa-se que no período do ensaio, as precipitações ficaram no limite mínimo de consumo, o que prejudicou a produtividade da cultura. Outro fator a ser considerado é que essa precipitação foi muito mal distribuída ao longo do período de condução do ensaio. Somando-se toda a precipitação ocorrida no período e dividindo-se pelo ciclo médio dos híbridos, obtém-se uma média diária de 4,43 mm (media de 3,79 $\mathrm{mm} \mathrm{dia}^{-1}$ em dezembro, 13,35 $\mathrm{mm} \mathrm{dia}^{-1}$ em janeiro, 0,2 $\mathrm{mm} \mathrm{dia}^{-1}$ em fevereiro, $1,65 \mathrm{~mm} \mathrm{dia}^{-1}$ em março e $0,0 \mathrm{~mm}$ dia $^{-1}$ em abril), o que fica abaixo das necessidades da cultura.

Tabela 8. Efeito dos herbicidas nicosulfuron e foramsulfuron + iodosulfuron methyl sodium na produtividade dos híbridos de milho testados. Marechal Cândido Rondon/PR, 2004/2005.

\begin{tabular}{|c|c|c|c|c|c|c|c|}
\hline \multirow{2}{*}{ Tratamentos } & \multirow{2}{*}{$\begin{array}{c}\text { Dose } \\
\left(\mathrm{g} \text { i.a. } \text { ha }^{-1}\right)\end{array}$} & \multicolumn{6}{|c|}{ Produtividade $\left(\mathrm{kg} \mathrm{ha}^{-1}\right)$} \\
\hline & & AS 32 & S2 709 & AS 1533 & AS 1545 & AS 1548 & AS 3466 \\
\hline 1. Testemunha & - & $2200,40 a^{*}$ & $2603,77 \mathrm{a}$ & $3156,45 \mathrm{a}$ & $2446,37 \mathrm{a}$ & $2396,47 \mathrm{a}$ & $3050,86 \mathrm{a}$ \\
\hline 2. Forams. + Iodos. & $22,5+1,5$ & $2197,48 \mathrm{a}$ & $2805,20 \mathrm{a}$ & $2880,48 \mathrm{a}$ & $2764,83 \mathrm{a}$ & $2312,75 \mathrm{a}$ & $2753,38 \mathrm{a}$ \\
\hline 3. Forams. + Iodos. & $45,0+3,0$ & $2371,10 \mathrm{a}$ & $2741,48 \mathrm{~b}$ & $3175,73 \mathrm{a}$ & $2783,63 \mathrm{a}$ & $2653,58 \mathrm{a}$ & 2367,18 a \\
\hline 4. Forams. + Iodos. & $90,0+6,0$ & $2406,03 \mathrm{a}$ & $2450,20 \mathrm{~b}$ & $2337,00 \mathrm{~b}$ & $1924,70 \mathrm{a}$ & $2333,00 \mathrm{a}$ & $2915,95 \mathrm{a}$ \\
\hline 5. Nicosulfuron & 30,0 & $2496,40 \mathrm{a}$ & $2623,77 \mathrm{a}$ & $2817,13 \mathrm{a}$ & $2723,75 \mathrm{a}$ & $2780,73 \mathrm{a}$ & 2726,03 a \\
\hline 6. Nicosulfuron & 60,0 & $2067,58 \mathrm{a}$ & $3151,18 \mathrm{a}$ & $2995,08 \mathrm{a}$ & $2912,70 \mathrm{a}$ & $2587,00 \mathrm{a}$ & $2580,38 \mathrm{a}$ \\
\hline 7. Nicosulfuron & 120,0 & $2861,18 \mathrm{a}$ & $3022,45 \mathrm{a}$ & $2663,30 \mathrm{~b}$ & $2820,88 \mathrm{a}$ & $2206,08 \mathrm{a}$ & $3079,20 \mathrm{a}$ \\
\hline $\mathrm{F}$ & - & $2,088^{\mathrm{NS}}$ & $5,125^{*}$ & $3,106^{*}$ & $1,877^{\mathrm{NS}}$ & $1,341^{\mathrm{NS}}$ & $1,834^{\mathrm{NS}}$ \\
\hline C.V. (\%) & - & 17,023 & 13,607 & 13,061 & 21,203 & 16,371 & 15,234 \\
\hline
\end{tabular}

Fonte: *Médias seguidas de mesma letra, na coluna, não diferem estatisticamente entre si, pelo teste de Agrupamento de SkottKnott. 
Observa-se que o herbicida foramsulfuron + iodosulfuron methyl sodium influenciou negativamente a produtividade do híbrido de milho S2 709, quando aplicado nas duas maiores doses (Tabela 8). Para o híbrido AS 1533, o herbicida foramsulfuron + iodosulfuron methyl sodium constatou-se influência apenas na maior dose testada.

$\mathrm{O}$ herbicida nicosulfuron influenciou negativamente a produtividade apenas do híbrido AS 1533, quando aplicado na sua maior dose.

Para o híbrido S2 709, o provável fator que influenciou na diminuição da produtividade foi a massa das espigas e para o híbrido AS 1533 foi o número de grãos por espiga.

Os dados obtidos no presente trabalho, confirmam as observações obtidas por DAMIÃO FILHO; MORO; TAVEIRA (1996); GREEN; ULRICH (1994); GREEN (1998); NEWHOUSE; WANG; ANDERSON (1991); MONKS; MULLINS; JOHSON (1992); PEREIRA FILHO; OLIVEIRA; PIRES (2000); FAHL; CARELLI (1997); MORTON; HARVEY (1992); LÓPEZ-OVEJERO; FANCELLI; DOURADO-NETO (2003); MORO; DAMIÃO FILHO (1999); ZAGONEL (2003), de que existe uma tolerância diferencial entre híbridos de milho aos herbicidas do grupo das sulfoniluréias. Confirmam também, a necessidade de testes de seletividade específicos para cada híbrido de milho, pois se comprova mais uma vez a existência de tolerância diferencial de cultivares de milho aos herbicidas do grupo das sulfoniluréias. Dessa forma, o potencial de uso e registro desses herbicidas devem ser restritos a determinados cultivares que tolerem o produto.

\section{Conclusões}

Pelos resultados obtidos e nas condições em que o experimento foi realizado, conclui-se que:

Os híbridos de milho testados possuem tolerância diferencial aos herbicidas nicosulfuron e foramsulfuron + iodosulfuron methyl sodium, sendo que as maiores doses aplicadas causaram maiores injúrias às plantas;

Os híbridos EXP S2 709 e AS 1533 foram os que apresentaram maior sensibilidade aos herbicidas aplicados, com reduções significativas de produtividade.

\section{Referências}

ANDREI, E. Compêndio de defensivos agrícolas. São Paulo: Organização Andrei, 2005. 1141p.

CARVALHO, F. T.; GALLI, A. J. B. Nova formulação de alachlor na seletividade e no controle de plantas daninhas na cultura do milho. In: CONGRESSO BRASILEIRO DE HERBICIDAS E PLANTAS DANINHAS, 19., 1993, Londrina. Resumos... Londrina: SBHED, 1993. p. 131.

CARVALHO, F. T.; PERUCHI, M.; PALAZZO, R. R. B. Eficácia de herbicidas no controle, em pós-emergência, de plantas daninhas na cultura do milho. Herbicidas, v. 2, n. 3, p. 143-147, 2001.

COMPANHIA NACIONAL DE ABASTECIMENTO CONAB. Disponível em: <http://www.conab.gov.br>. Acesso em: 18 out. 2009.

CONSTANTIN, J.; OLIVEIRA JUNIOR., R. S.; MACIEL, C. D. G.; ROSSI, J. M. Utilização de isoxaflutole+atrazina para o manejo de plantas daninhas em milho. In: CONGRESSO BRASILEIRO DA CIÊNCIA DAS PLANTAS DANINHAS, 22., 2000, Foz do Iguaçu. Resumos... Londrina: SBCPD, 2000. p. 280.

DAMIÃO FILHO, C. F.; MÔRO, F. V.; TAVEIRA, L. R. Respostas de híbridos de milho ao nicosulfuron. I Aspectos biológicos e na produção. Planta Daninha, Viçosa, v. 14, n. 1, p. 3-13, 1996.

EMPRESA BRASILEIRA DE PESQUISA AGROPECUÁRIA - EMBRAPA. Centro Nacional de Pesquisa de Solos. Sistema brasileiro de classificação de solos: 4a aproximação. Rio de Janeiro, EMBRAPA, 1999. $169 \mathrm{p}$.

EMPRESA BRASILEIRA DE PESQUISA AGROPECUÁRIA - EMBRAPA. Centro Nacional de Pesquisa de Solos. Disponível em: $<$ http://www.cnpms. embrapa.br>. Acesso em: 11 de jul. 2004.

EMBRAPA MILHO E SORGO - EMBRAPA. Cultivo do milho. Sistemas de produção. 2008. Disponível em: $<$ http://www.cnpms.embrapa.br/publicacoes/milho/ index.htm.>. Acesso em: 27 set. 2009. 
EUROPEAN WEED RESEARCH COUNCIL - EWRC. Committee of methods in weed research. Oxford : 1964. v. 4, p. 88. (Report of the 3 and 4 Meetings).

FAHL, J. T.; CARELLI, M. L. C. Eficiência do nicosulfuron no controle do capim-massambará na cultura do milho. Planta Daninha, Viçosa, v. 15, n. 1, p. 46-52, 1997.

GREEN, J. M. Differential tolerance of corn (Zea mays) inbreds to four sulfonylurea herbicides and bentazon. Weed Technology, Lawrence, v.12, p. 474-477, 1998.

GREEN, J. M.; ULRICH, J. F. Response of maize (Zea mays) inbreds and hybrids to nicosulfuron. Pestic. Sci., v. 40, p. 187-191, 1994.

KARAM, D., MELHORANÇA, A. L. Plantas daninhas. Sistemas de produção de milho. In: CRUZ, J. C., VERSIANI, R. P., FERREIRA, M. T. R. (Ed.). Sete Lagoas, 2000. EMBRAPA-CNPMS. Disponível em: $<$ http:// www.cnpms.embrapa.br $>$. Acesso em: 12 ago. 2003.

LOPEZ-OVEJERO, R. F., FANCELLI, A. L., DOURADO-NETO, D. Seletividade de herbicidas para a cultura de milho (Zea mays) aplicados em diferentes estádios fenológicos da cultura. Planta Daninha. set./ dez. 2003, v. 21, n. 3, p.413-419. Disponível em: <http:// www.scielo.br>. Acesso em: 11 jul. 2004.

LORENZI, H. Manual de identificação e controle de plantas daninhas. 5. ed. Nova Odessa : Instituto Plantarum, 2000.

MONKS, D. W.; MUllinS, C. A.; JOHSON, K. E. Response of sweet corn (Zea mays) to nicosulfuron and primsulfuron. Weed Technology, Champaign, v. 6, p. 280-283, 1992.

MÔRO, F. V.; DAMIÃO FILHO, C. F. Alterações morfoanatômicas das folhas de milho submetidas à aplicação de nicosulfuron. Planta Daninha, Viçosa, v. 17, n. 3, p. 331-337, 1999.

MORTON, C. A.; HARVEY, R. G. Sweet corn (Zea mays) hybrid tolerance to nicosulfuron. Weed Technology, Champaign, v. 6, p. 91-96, 1992.

NEWHOUSE, K. E.; WANG, T.; ANDERSON, P. C. Imidazolinone-resistant crops. In: SHANER, D. L.; O' CONNOR, S. L. (Eds.). The imidazolinone herbicides. Boca Raton: CRC Press, 1991. p. 139-150.

PEREIRA FILHO, I. A., OLIVEIRA, M. F., PIRES, N. M. Tolerância de híbridos de milho ao herbicida nicosulfuron. Planta Daninha, Viçosa, v. 18, n. 3, p. 479482, 2000.
PINTO, J. J. O.; SPERANDIO, C. A.; FERREIRA, F. B.; LAMEGO, F. P. Controle de plantas daninhas na cultura do milho com herbicidas de aplicação em pré e pós-emergência. In: CONGRESSO BRASILEIRO DA CIENCIA DAS PLANTAS DANINHAS. 2000, Foz do Iguaçu. Resumos... Foz do Iguaçu: SBCPD, 2000. p. 279.

RODRIGUES, B. N; ALMEIDA, F. S. Guia de herbicidas. 4 ed. Londrina: Ed. dos Autores. 1998. 648p.

ROSSI, I. H.; OSUNA, J. A.; ALVES, P. L. C. A.; BEZUTT, A. J. Interferência das plantas daninhas sobre algumas características agronômicas e a produtividade de sete cultivares de milho. Planta Daninha, Botucatu, v. 14. n. 2, p. 134-148, 1996.

SILVA, A. A.; MELHORANÇA, A. L. Controle de plantas daninhas na cultura do milho. In: Empresa Brasileira de Pesquisa Agropecuária. UEPAE - Dourados. Milho: informações técnicas. Dourados, 1991. p. 114-127. (Circular Técnica, n. 20).

SILVA, J. B.; RODRIGUES, M. A. T.; BEGLIOMINI, E. Indicações para o controle de plantas daninhas na cultura do milho em pós-emergência com o herbicida Sanson 40 $\mathrm{SC}$ e sua mistura $1+2$ com atrazine. O Ruralista, Belo Horizonte, v. 35, n. 440, p. 9-11, 1998.

SILVA, M. S. J.; ASMUS, G. L.; CORREA, A. M. Estudo do herbicida nicosulfuron no controle de plantas daninhas na cultura do milho (Zea mays L.). In: CONGRESSO BRASILEIRO DE HERBICIDAS E PLANTAS DANINHAS, 19., 1993, Londrina. Resumos... Londrina: SBHED, 1993. p. 148-149.

SOCIEDADE BRASILEIRA DA CIÊNCIA DAS PLANTAS DANINHAS. Procedimentos para instalação, avaliação e análise de experimentos com herbicidas. Londrina: SBCPD, 1995. 42p.

VELINI, E. D.; FREDERICO, L. A.; BICUDO, S. J.; ANTUNIASSI, U. R. Eficiência de herbicidas pré e pós emergentes iniciais recomendados para a cultura do milho, no controle de plantas daninhas e avaliação de efeitos dos mesmos sobre a produtividade da cultura. In: CONGRESSO BRASILEIRO DE HERBICIDAS E PLANTAS DANINHAS, 19., 1993, Londrina. Resumos... Londrina: SBHED, 1993. p. 41-142.

ZAGONEL, J. Equip Plus - eficiência comprovada. Cultivar Grandes Culturas, Pelotas, n. 46, p. 30-32, 2003. 